\title{
Descumprimento do Código de Ética Odontológica em redes sociais - análise de irregularidades vinculadas à publicidade e propaganda
}

\author{
Noncompliance with the Code of Ethical Dentistry in social networks - analysis of \\ irregularities linked to advertising and propaganda
Incumplimiento del Código de Odontología Ética en las redes sociales. Análisis de irregularidades vinculadas a la publicidad y la propaganda.

\begin{abstract}
Karolyne de Melo Soares ${ }^{1 *}$, Ana Karina de Almeida Rolim ${ }^{2}$, Diego Filipe Bezerra da Silva ${ }^{2}$, Cristiane Araújo Maia Silva ${ }^{3}$, Fernanda de Araújo Trigueiro Campos ${ }^{4}$, Moises Willians da Silva ${ }^{5}$, Maria Regina Macedo-Costa ${ }^{6}$, Carlus Alberto Oliveira dos Santos².
\end{abstract}

\section{RESUMO}

Objetivo: Investigar as irregularidades éticas em Odontologia vinculadas a publicidade e propaganda em redes sociais (Facebook ${ }^{\circledR}$ e Instagram ${ }^{\circledR}$ ). Métodos: Trata-se de um estudo observacional transversal e exploratório. Os dados foram coletados por meio de um formulário elaborado a partir de itens obrigatórios do Código de Ética Odontológica-CEO, Resolução CFO-118/2012. A avaliação teve uma amostra de 133 perfis, sendo 52 situados na rede social Facebook ${ }^{\circledR}$ e 81 presentes na rede social Instagram $\AA$ no período de maio a junho de 2019, coletadas e avaliadas por um único pesquisador. Os dados foram tabulados no programa Microsoft Excel® e analisados descritivamente em SPSS 21.0. Resultados: Dentre as infrações éticas mais frequentes, estão a não exibição do número de inscrição da pessoa física ou jurídica no Conselho Regional de Odontologia por 101 perfis e a não identificação do responsável técnico por 130 perfis. Além disso se mostraram frequentes o número de páginas ilegais, composto por 48 perfis, não pertencentes a profissionais da Odontologia, realizando vendas/anúncios de produtos odontológicos. Conclusão: A maioria das propagandas e publicidades não seguem as normas exigidas pelo CEO e há necessidade de fiscalização das redes sociais.

Palavras-chave: Odontologia, Redes Sociais, Código de ética.

\begin{abstract}
Objective: To investigate ethical irregularities in dentistry linked to advertising and propaganda in social networks (Facebook ${ }^{\circledR}$ and Instagram $\AA$ ). Methods: This is a cross-sectional and exploratory observational study. The data were collected through a form elaborated from mandatory items of the Code of Dental EthicsCEO, Resolution CFO-118/2012. The evaluation had a sample of 133 profiles, being 52 located in the social network Facebook ${ }^{\circledR}$ and 81 present in the social network Instagram ${ }^{\circledR}$ from May to June of 2019, collected and evaluated by a single researcher. The data were tabulated in the Microsoft Excel® program and analyzed descriptively in SPSS 21.0. Results: Among the most frequent ethical infractions are the non-display of the registration number of the individual or legal entity in the Regional Council of Dentistry for 101 profiles and the

\footnotetext{
1 Instituto de Educação Superior da Paraíba (IESP), Cabedelo-PB.*E-mail: karolmelosoaresjp@gmail.com

2 Universidade Estadual da Paraíba (UEPB), Campina Grande - PB.

3 Universidade Federal da Paraíba (UFPB), João Pessoa-PB.

${ }^{4}$ Universidade Cruzeiro do Sul (Unicsul), São Paulo-SP.

${ }^{5}$ Centro Universitário de João Pessoa - UNIPÊ, João Pessoa-PB.

${ }^{6}$ Universidade Federal do Rio Grande do Norte (UFRN), Rio Grande do Norte-RN.
} 
non-identification of the technical responsible for 130 profiles. In addition, the number of illegal pages was made up of 48 profiles, not belonging to dental professionals, making sales / announcements of dental products. Conclusion: Most advertisements and advertisements do not follow the standards required by the CEO and there is a need for social media oversight.

Keywords: Dentistry, Social Networks, Code of Ethics.

\section{RESUMEN}

Objetivo: Investigar las irregularidades éticas en odontología relacionadas con la publicidad y la propaganda en las redes sociales (Facebook ${ }^{\circledR}$ e Instagram $\AA$ ). Métodos: Este es un estudio observacional transversal y exploratorio. Los datos se recopilaron a través de un formulario elaborado a partir de los puntos obligatorios del Código de ética dental-CEO, Resolución CFO-118/2012. La evaluación tenía una muestra de 133 perfiles, de los cuales 52 estaban ubicados en la red social Facebook $\AA^{\circledR}$ y 81 en la red social Instagram ${ }^{\circledR}$ de mayo a junio de 2019, recopilados y evaluados por un solo investigador. Los datos se tabularon en el programa Microsoft Excel $\circledast$ y se analizaron de manera descriptiva en SPSS 21.0. Resultados: entre las infracciones éticas más frecuentes se encuentran la no visualización del número de registro de la persona física o jurídica en el Consejo Regional de Odontología para 101 perfiles y la no identificación del técnico responsable de 130 perfiles. Además, el número de páginas ilegales estaba compuesto por 48 perfiles, no pertenecientes a profesionales dentales, que realizan ventas / anuncios de productos dentales. Conclusión: La mayoría de los anuncios y publicidades no siguen los estándares exigidos por el CEO y existe una necesidad de supervisión de las redes sociales.

Palabras clave: Odontología, Redes Sociales, Código de Ética.

\section{INTRODUÇÃO}

Devido ao grande número de Cirurgiões-Dentistas (CD's) espalhados pelo Brasil e mundo, a competitividade no âmbito profissional é notória. Visando a melhoria de atuação no mundo do trabalho, os CD's têm investido em ferramentas que os ajudem na busca e manutenção de clientes/pacientes (LIMA AIC, et al., 2016).

O Marketing, na prática odontológica, constitui um importante instrumento para atrair e fidelizar pacientes, sem ferir o código de ética da profissão, com o objetivo de estreitar a relação paciente/profissional e, além disso éserá um grande divulgador do trabalho (MARTINS ALG, et al., 2011; PARANHOS LR, et al., 2011).

Diante de várias estratégias de marketing disponíveis, a mais frequentemente utilizada é a publicidade e propaganda externa, por meio de cartões, placas, folhetos e da internet, que tem liderado dentre formas de veiculação escolhidas pelos CD's (FELíCIO BC, et al., 2013).

As redes sociais tem sido utilizada como ferramentas para atingir o público-alvo desejado, com o objetivo de divulgação dos serviços e produtos. $O$ uso da internet tem se mostrado uma estratégia simples, barata, eficaz e amplamente utilizada para propaganda (LIMA AIC, 2016; MIRANDA GE, et al., 2013).

É necessário cautela quando o assunto é publicidade e propaganda. Toda veiculação deve estar dentro dos padrões exigidos pelo Código de Ética Odontológica (CEO), que é um instrumento elaborado para orientar a conduta dos CD's sobre os aspectos éticos da profissão, e mesmo assim, ainda existe um número alarmante de profissionais que não o seguem (FELícIO BC, et al., 2013; LIMA AIC, et al., 2016)

Garcia SJ e Caetano JC (2008) apontaram em seu estudo que a infração ética mais comumente praticada pelos CD's é a propaganda irregular ou ilegal, constituído de $57,14 \%$. Salienta-se tambem que, na internet, é possível encontrar usuários não habilitados realizando revenda e promoções de produtos odontológicos sem prescrição profissional, descumprindo $\mathrm{CEO}$, desvalorizando a classe profissional e, principalmente, colocando em risco à saúde dos usuários. 
Nessa perspectiva, objetiva-se com este estudo investigar, por meio de buscas nas redes sociais (Facebook $\AA$ e Instagram $\AA$ ), as irregularidades éticas correlacionadas às publicidades e propagandas no âmbito da Odontologia.

\section{MÉTODOS}

Trata-se de um estudo observacional transversal, realizado através de um instrumento adaptado do estudo de Lima AIC et al. (2016), composto por 13 perguntas sobre a utilização do marketing na Odontologia, com elaboração baseada nas infrações éticas dispostas no CEO.

O cálculo amostral foi realizado baseado no estudo de Miranda GE et al. (2013), utilizando-se um erro tipo I de $5 \%$ e um erro tipo II de $20 \%$ e que, considerando a impossibilidade da obtenção do universo de perfis de interesse, obteve-se uma amostra de 133 perfis.

Para coleta e análise dos dados de propagandas ilegais e irregulares, realizou-se a busca de perfis nas redes sociais de forma sistemática e não-sistemática, através da ferramenta de busca disponível em cada aplicativo. Foram incluídos os perfis que eram de acesso público, que tinham relação com a odontologia e os critérios de exclusão foram perfis estrangeiros, duplicados e privados.

Os descritores utilizados para o Facebook $®$ foram: "Consultório Odontológico", "Clareamento dental", "Prótese Dentária”, "Ortodontia”. Para o Instagram®: "Consultório Odontológico", "Odontologia Especializada", "Clareamento Dental", "Ortodontia" e "Prótese dentária".

Após a busca, cada perfil foi analisado de acordo com as perguntas do instrumento. No Facebook®, a busca foi efetuada na seção "Marketplace", num raio de 100 quilômetros a partir da cidade João Pessoa, Paraíba, Brasil, e no campo de busca geral, avaliando-se a descrição da publicação, linguagem utilizada, identificação do perfil (nome completo, registro) e fotos da galeria. No Instagram $\AA$ as buscas foram realizadas na aba de busca no "explorar", avaliando-se biografia, fotos e descrição.

Um mesmo pesquisador avaliou os perfis e transferiu os dados para a plataforma do Excel®. A coleta de dados foi realizada de maio a junho de 2019.

Os dados coletados foram digitados no banco de dados Excel ${ }^{\circledR}$ e transferidos e analisados descritivamente no software estatístico SPSS versão 21.0.

Por se tratar de um estudo que coleta informações de perfis públicos nas redes sociais, conforme interpretado no trabalho de Lima AIC et al. (2016), este estudo não foi submetido à apreciação ao Comitê de Ética em Pesquisa (CEP).

\section{RESULTADOS}

Foram encontrados $n=133$ perfis, sendo $n=52$ situados na rede social Facebook ${ }^{\circledR}$ e $n=81$ presentes na rede social Instagram $\AA$. Os perfis com postagens ilegais e irregulares, foram avaliados de acordo com as 13 perguntas baseadas no Código de Ética Odontológica (Tabela 1).

No presente estudo, verificou-se que nas duas redes sociais, mais da metade dos perfis não exibem o número de inscrição da pessoa física ou jurídica, constando no CEO item obrigatório para fins de divulgação, obtendo um total de $76 \%$ dos perfis que não seguem essa norma.

Os perfis identificados como de clínicas odontológicas, ou seja, perfis jurídicos, quanto a exibição da inscrição do responsável técnico dos perfis analisados, $98 \%$ não exibiam o registro no $\mathrm{CRO}$ em suas respectivas páginas, sendo tambem item obrigatório, com base no CEO.

Quanto a divulgação de preços, ainda foi constatado que 14\% dos perfis avaliados exibiram tanto preços em suas publicações quanto formas de pagamento e/ou bandeiras de cartão de crédito, que implicam comercialização da Odontologia, contrariando o CEO e constituindo-se infração ética. 
Tabela 1 - Quantitativo de perfis irregulares com o Código de Ética Odontológica.

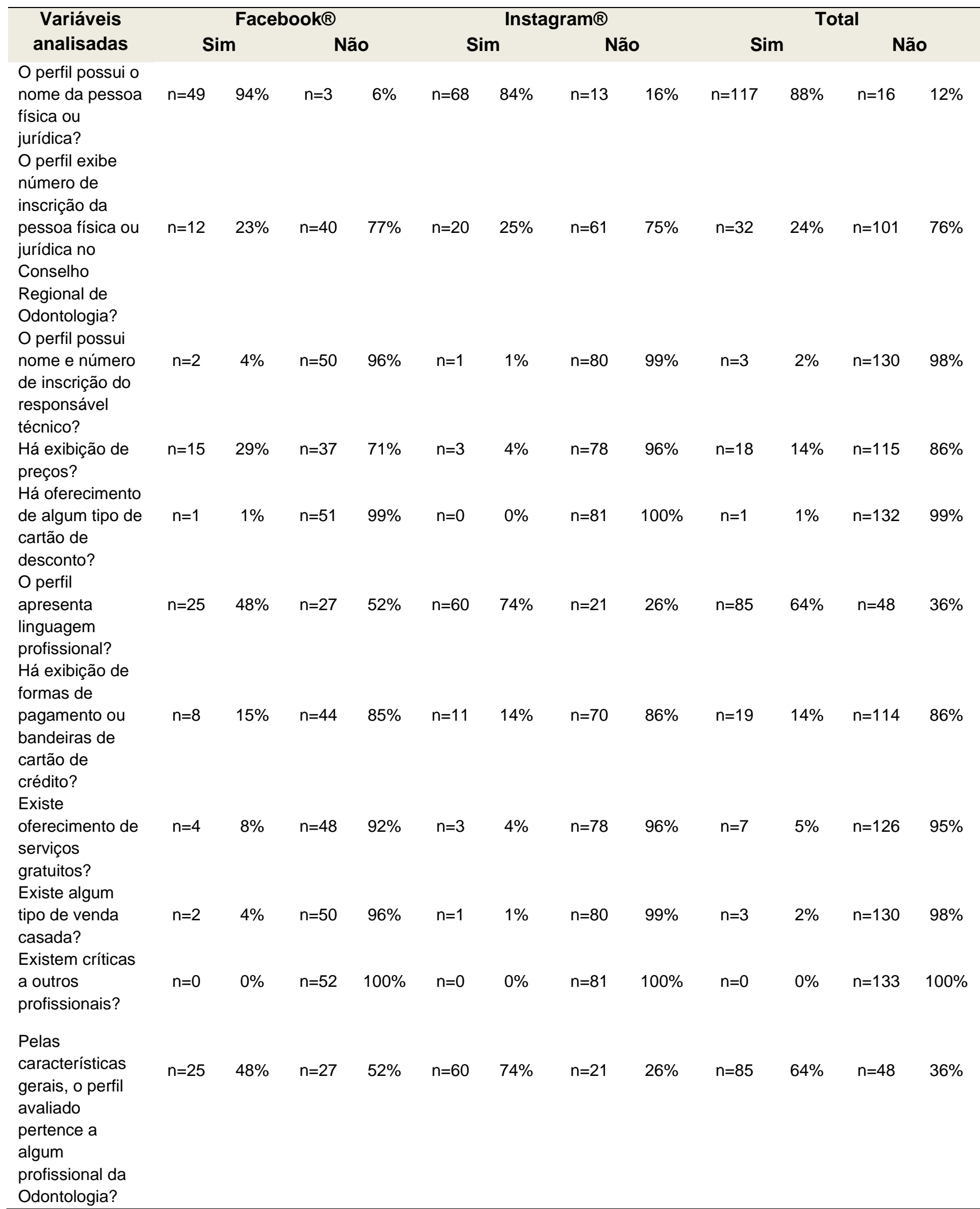

Fonte: Soares KM, Rolim AKA, Silva DFB, 2019. 
Dos perfis avaliados, 48 deles (36\%) não pertenciam a profissionais da Odontologia, dos quais, todos estes realizavam venda/anúncio de materiais ou procedimentos de exclusividade odontológica (Tabela 2).

A Prótese Dentária foi o material mais comercializados em ambas as redes sociais. Quando se trata de especialidade, a mais relacionada no Facebook é a Ortodontia, com $24 \%$. Todavia, no Instagram, a especialidade de maior destaque é a Prótese Dentária.

Tabela 2 - Frequência relativa e absoluta de perfis ilegais que realizam vendas de produtos odontológicos.

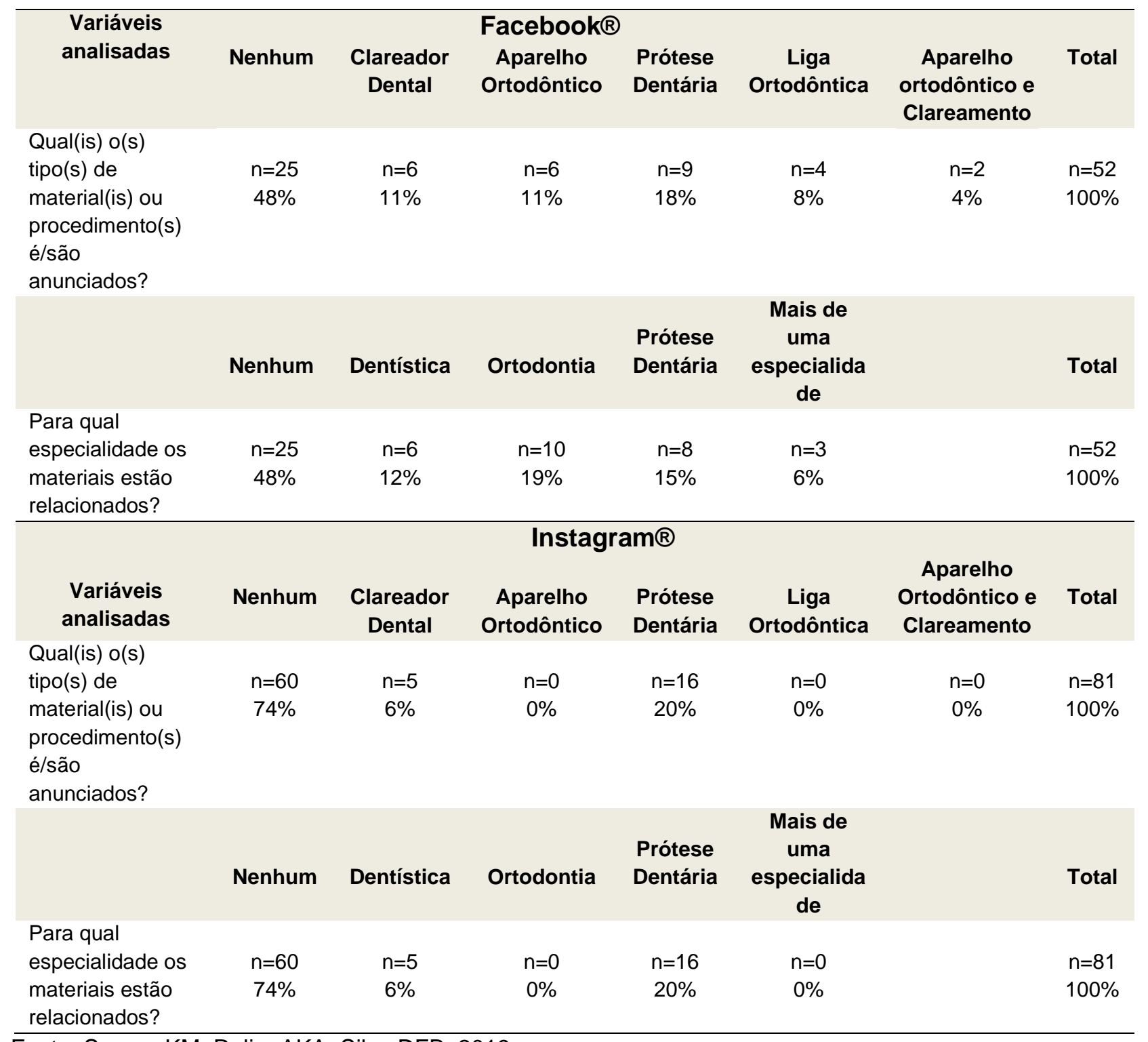

Fonte: Soares KM, Rolim AKA, Silva DFB, 2019.

\section{DISCUSSÃO}

A importância desse trabalho, dar-se como meio de divulgação do panorama atual das publicações vinculadas aos serviços odontológicos encontrados em redes sociais. Sabe-se que o uso das técnicas de marketing são aceitáveis no ramo odontológico, mas de forma que não haja desvalorização da profissão. Com isso, o Código de Ética Odontológica (CEO) estabeleceu regras relacionadas as práticas publicitárias, com o intuito de constituir padrões para um bom desenvolvimento da profissão (MARTINS ALG et al., 2011; FELíCIO BC et al., 2013). 
De acordo com o CEO, artigo 43, "é obrigatório constar na comunicação e divulgação, o nome, o número de inscrição da pessoa física ou jurídica, bem como nome representativo. No caso de pessoas jurídicas, também o nome e o número de inscrição do responsável técnico". O fato de $76 \%$ neste estudo não exibirem o registro nos perfis, é semelhante ao que foi descrito por Lima AIC et al. (2016) ao analisar 257 perfis, onde $85,2 \%$ deles não exibiam o registro no $\mathrm{CRO}$ e $86,2 \%$ dos perfis jurídicos não exibiam identificação do responsável técnico. Segundo o inciso I do artigo 44 do CEO, constitui infração ética divulgar preços, serviços gratuitos, modalidades de pagamento ou outras formas, implicando a comercialização da Odontologia (CFO, 2012). Porém, cerca de $14 \%$ dos perfis encontrados neste estudo, ainda divulgam preços e modalidades de pagamento. Já no estudo de Serra et al. (2005) verificaram que $4,10 \%$ dos respondentes divulgam modalidades de pagamento e $2,56 \%$ preços.

Miranda GE et al. (2013), afirmam que apenas 5,99\% faziam esse tipo de anúncio e em nenhum deles observou-se preços, apenas modalidades de pagamento (bandeiras de cartão de crédito, parcelamento de pagamento) ou expressão do tipo "orçamento gratuito", que constitui infração ética divulgar/oferecer diagnósticos gratuitos. Corroborando, Malheiros ZM (2011) em seu estudo, encontrou que 5\% das clínicas odontológicas em Websites divulgaram preços ou formas de pagamento.

No parágrafo 1 do artigo 41 do CEO, é vedado aos Técnicos de Prótese Dentária, bem como laboratórios de prótese dentária realizarem propagandas ou publicidades dirigida ao público em geral (CFO, 2012). Viola, Oliveira e Dotta (2011) afirmam que é de inteira responsabilidade do profissional acerca dos anúncios veiculados. Neste estudo, perfis identificados como de técnicos em prótese dentária comercializavam peças protéticas, obtendo $18 \%$ dessa comercialização no Facebook ${ }^{\circledR}$ e $20 \%$ no Instagram $\AA$, alcançando destaque com relação a outros produtos, constituindo infração ética. Além da prótese dentária ser comercializada irregularmente, $36 \%$ dos perfis irregulares e não pertencentes a profissionais da odontologia realizavam venda/anúncio de materiais ou procedimentos de exclusividade odontológica. Clareador dental, liga e fios de aparelho ortodôntico e procedimento de instalação dos produtos foram encontrados através deste estudo. Para tanto, essas veiculações caracterizam conduta inadequada frente ao CEO e devem ser reprimidas pelos Conselhos Regionais de Odontologia.

Quando se trata de especialidade, a mais relacionada no Facebook é a Ortodontia, com 24\%. Todavia, no Instagram, a especialidade da Prótese Dentária é a mais relacionada. O CEO caracteriza como infrações éticas a propaganda enganosa; a mercantilização da Odontologia e outras condutas que sejam relacionadas ao aviltamento da profissão. A função do CEO não é apenas para estabelecer regras padronizadas dentro de uma categoria profissional, mas também defender/amparar todos os profissionais e pessoas envolvidas no exercício da profissão (BRASIL, 2012; FELíCIO BC et al., 2013).

Sendo assim, o marketing é de indiscutível importância para a solução dos problemas de competitividade da classe odontológica, contudo, a forma como ele está sendo utilizado mediante as regulamentações existentes, tem provocado preocupações. Por isso, para que a profissão seja valorizada, cabe aos profissionais saberem dos limites éticos (PARANHOS LR et al., 2011; FELÍCIO BC et al., 2013).

Face ao exposto, esses resultados refletem que existe a necessidade de conscientização de parte dos profissionais quanto aos meios de comunicação e divulgação de seus serviços de forma ética. Ações imprescindíveis devem ser realizadas por parte dos órgãos e instituições responsáveis pelas fiscalizações, afim de reduzir as infrações já existentes.

\section{CONCLUSÃO}

A maioria dos perfis analisados não seguem as normas exigidos pelo Código de Ética Odontológica. Portanto, se faz necessário uma fiscalização ante a perfis irregulares e tambem aos ilegais, de usuários que não são profissionais da odontologia, que comercializam produtos odontológicos apenas de uso profissional e que no âmbito universitário seja reforçado a existência do CEO e a obrigação do cumprimento das normas, para que as infrações observadas neste estudo não se repitam. Além disso, identifica-se uma carência sobre a abordagem desse assunto na literatura, caracterizando uma limitação deste estudo. 


\section{REFERÊNCIAS}

1. ARCIER NM, et al. A importância do marketing odontológico para enfrentar um mercado competitivo. Revista Odontológica de Araçatuba, 2007; 28(3): 24-32.

2. BRASIL. Conselho Federal de Odontologia. Resolução CFO n 118, de 11 de maio de 2012. Revoga o Código de Ética Odontológica aprovado pela resolução CFO-42/2003 e aprova outro em substituição.Diário Oficial [da] União, Brasília, DF, 14 jun. 2012

3. CONSELHO FEDERAL DE ODONTOLOGIA. Resolução № 118, de 11 de maio de 2012. Revoga o Código de Ética Odontológica aprovado pela Resolução CFO-42/2003 e aprova outro em substituição.

4. FELÍCIO BC, et al. Ética no marketing odontológico. Revista do CROMG, 2013; 14(2): 28-32.

5. GARCIA SJ, CAETANO JC. O código de ética odontológica e suas infrações: um estudo sobre os processos éticosprofissionais dos cirurgiões dentistas do estado de Santa Catarina. Revista Odontologia Clínico-científica, 2008; 7(4): 307-13.

6. LIMA AIC, et al. Análise dos perfis de clínicas odontológicas e de Cirurgiões-dentistas em duas redes sociais quanto aos aspectos éticos, de propaganda e publicidade. Revista Brasileira de Odontologia Legal, 2016; 3(2): 66-73.

7. MALHEIROS ZM. Avaliação da qualidade da informação em websites de clínicas e consultórios odontológicos. Dissertação [Mestrado em Odontologia]. USP, São Paulo, 2011; 140 p.

8. MARTINS ALG, et al. Avaliação dos aspectos éticos da publicidade e propaganda odontológica divulgadas por profissionais em São Luís - MA. Revista de Pesquisa em Saúde, 2011; 12(2): 23-26.

9. MIRANDA GE, et al. Análise de websites de cirurgiões-dentistas quanto aos aspectos éticos e legais relativos à publicidade e propaganda. Revista brasileira de odontologia, 2013; 70(1): 80-4.

10. PARANHOS L, et al. Implicações éticas e legais do marketing na Odontologia. Revista RSBO 2011; 8(2):219-24.

11. SERRA MC, et al. Ferramentas de marketing empregadas por cirurgiões dentistas. Revista Gaúcha de Odontologia RGO 2005; 53(2):85-164.

12. VIOLA NV; OLIVEIRA ACM; DOTTA EAV. Marketing in dentistry: a tool that makes the difference. Revista Brasileira de odontologia $2011 ; 68(2): 248-251$. 